\title{
VEGFR2 Expression in Head and Neck Squamous Cell Carcinoma Cancer Cells Mediates Proliferation and Invasion
}

\author{
Hui-Min $\mathrm{Xu}^{1 *}$, Jian-Guo Zhu ${ }^{1}$, Lian $\mathrm{Gu}^{1}$, Song-Qun $\mathrm{Hu}^{2}$, Hao $\mathrm{Wu}^{2}$
}

\begin{abstract}
Vascular endothelial growth factor 2 (VEGFR2) was initially identified as a receptor of VEGF on endothelial cells with a role in regulating angiogenesis during organism development and tumorigenesis. Previously, in cancer tissue, VEGFR2 has been reported to be expressed in endothelial cells. In our research, we found that VEGFR2 was expressed not only in endothelial cells but also cancer cells in head and neck squamous cell carcinomas (HNSCCs). Knockdown of VEGFR2 in Hep2 cells could arrest the cell cycle in G0/G1, leading to a decrease in proliferation. We also present evidence that MAPK/ERK signal pathways and expression of CDK1 downstream of VEGFR2 might regulate proliferation and cell cycle arrest. Furthermore, we discovered that down-regulate VEGRF2 in Hep2 cells could significantly affect the invasion ability. Taken together, our data suggest that VEGFR2 might regulate proliferation and invasion in HNSCC cancer cells in vivo.
\end{abstract}

Keywords: HNSCC - VEGFR2 - proliferation - invasion

Asian Pac J Cancer Prev, 17 (4), 2217-2221

\section{Introduction}

Head and neck squamous cell carcinoma (HNSCC), which is diagnosed 500,000 new cases every year, is ranked among the 10 most common cancers worldwide (Rothenberg and Ellisen, 2012; Das et al., 2014). Due to the large amount of people consuming cigarettes and severely air pollution in China, it is much higher risk to suffer from HNSCC in China (Pathare et al., 2011). Nowadays, the therapies of HNSCC are surgical resection combined with radiotherapy and chemotherapy. However, the deficiency of biomarker for diagnosis and prognosis causes the low survival rate of HNSCC patients who diagnosed at advanced stages (Reya and Clevers, 2005; Rehman and Wang, 2009).

Vascular endothelial growth factor 2 (VEGFR2), also named as Kinase insert domain receptor (KDR), is a kind of type III receptor tyrosine kinase. It was first reported as the receptor of vascular endothelial growth factor (Parast et al., 1998). The activation of VEGFR2 leads to the proliferation, survival and permeability of endothelial cells via Raf/Mek/Erk, PI3K/Akt, and PI3K/Akt/nitric oxide pathways. Therefore, VEGFR2 is the key factor to mediate the angiogenesis during the process of development and tumorigenesis. So far, many drugs targeting VEFGFR2 has been developed and some of them work effectively (Gasparini et al., 2005; Krajewska et al., 2015). Recently, VEGFR2 has been reported to express in the tumor cells besides the endothelial cells and the expression levels are associated with prognosis (Brekken and Thorpe, 2001; Ye et al., 2004; Guo et al., 2010). There is no previous research detecting the expression of VEGFR2 in the tumor cells of HNSCC. In order to assess the relationship of VEGFR2 and the tumorigenesis of HNSCC, we preformed immunehistochemmical study on HNSCC tumors derived from 109 patients who undergone primary tumor resection in Affiliated Hospital of Natong University. We also examined the role of VEGFR2 in the proliferation and migration of Hep2 cancer cells which is a kind of human laryngeal carcinoma cell line.

\section{Materials and Methods}

\section{Patients and tissue samples}

The specimens of 109 tumor tissues and 28 paracarcinoma tissues were obtained from patients who underwent the surgery without previous chemotherapy and radiotherapy at ENT head and neck surgery department, Affiliated Hospital of Nantong University from January, 2002 to May, 2010. The HNSCC specimens were formalin-fixed paraffin-embedded. The specimen arrays were provided by Shanghai Outdo Biotech Co., Itd.

\section{Reagents and antibodies}

The following reagents and antibodies were used in this study: rabbit polyclonal anti- VEGFR2 (Abcam),

${ }^{1}$ Department of Otolaryngology-Head and Neck Surgery, Changshu No.1 People's Hospital Affiliated to Soochow University, Changshu, ${ }^{2}$ Department of Otolaryngology-Head and Neck Surgery, Affiliated Hospital of Nantong University, Nantong, China *For correspondence: 13921522245@163.com 


\section{Hui-Min Xu et al}

mouse monoclonal anti-CDK1 (Abcam), rabbit polyclonal anti-ACTIN (Abcam), rabbit polyclonal anti-P-ERK (Abcam), goat polyclonal anti-HA (Abcam). Z-LeuLeu-Leu-al (MG132) and Cycloheximide (CHX) were purchased from Sigma Aldrich.

\section{Western blot}

Tissue and cell proteins were immediately homogenized in a homogenization buffer containing $50 \mathrm{mM}$ Tirs- $\mathrm{HCl}$, PH 7.5, $150 \mathrm{mM} \mathrm{NaCl}, 0.1 \%$ NP-40, 5 mM EDTA, $60 \mathrm{mM}$ $\beta$-glycerophosphate, $0.1 \mathrm{mM}$ sodium orthovanadate, 0.1 $\mathrm{mM} \mathrm{NaF}$ and complete protease inhibitor cocktail (Roche Diagnostics), and then centrifuged at $12,000 \mathrm{~g}$ for $20 \mathrm{~min}$ to collect the supernatant. Protein concentrations were measured with a Bio-Rad protein assay (BioRad, Hercules, CA, USA). The supernatant diluted in $2 \times$ SDS loading buffer and boiled for $15 \mathrm{~min}$. Proteins were separated with SDS-polyacrylamide gel electrophoresis(SDS-PAGE) and then transferred to polyvinylidenedifluoride filter (PVDF) membranes (Millipore, Bedford, MA). The membranes were blocked with $5 \%$ fat-free milk in TBST $(150 \mathrm{mM}$ $\mathrm{NaCl}, 20 \mathrm{mM}$ Tris, $0.05 \%$ Tween-20) for $2 \mathrm{~h}$ at room temperature. Thereafter the membranes were washed with TBST three times and incubated overnight with the primary antibodies and later horseradish peroxidase-linked IgG as the secondary antibodies for $2 \mathrm{~h}$ at room temperature. The band density was determined by a computer-assisted image-analysis system (Adobe Systems, San Jose, CA) and normalized against ACTIN levels. Values were responsible for at least three independent reactions.

\section{Immunohistochemistry (IHC)}

Immunostaining was performed by using the avidin biotin peroxidase complex. The sections were deparaffinized with a graded ethanol series, and endogenous peroxidase activity was blocked by soaking in $3 \%$ hydrogen peroxide for $30 \mathrm{~min}$. Then, the sections were processed in $10 \mathrm{mmol} / \mathrm{L}$ citrate buffer $(\mathrm{PH}=6.0)$ and heated to $121^{\circ} \mathrm{C}$ in an autoclave for $20 \mathrm{~min}$ to retrieve the antigen. After rinsing in PBS $(\mathrm{PH}=7.2)$, the sections were then incubated with anti-VEGFR2 antibody (diluted $1: 200)$ for $2 \mathrm{~h}$ at room temperature. Negative control slides were processed in parallel using a nonspecific immunoglobulin IgG (Sigma Chemical Co., St. Louis, $\mathrm{MO})$ at the same concentration as the primary antibody. All slides were processed using the peroxidase-antiperoxidase method (DAKO, Hamburg, Germany). After rinsing in PBS, the peroxidase reaction was visualized by incubating the sections with the liquid mixture $(0.02 \%$ diaminobenzidinetetrahydrochloride, $0.1 \%$ phosphate buffer solution, and $3 \% \mathrm{H}_{2} \mathrm{O}_{2}$ ). After rinsing in water, the sections were counterstained with hematoxylin, dehydrated, and cover slipped.

\section{Cell cultures and transient transfection}

The human laryngeal carcinoma cell line Hep2 was purchased from the Shanghai Institute of Cell Biology, Academic Sinica and cultured in high-glucose DMEM (GibCo BRL, GrandIsland,NY, USA)supplemented with $20 \%$ fetal bovine serum, $100-\mathrm{U} / \mathrm{ml}$ penicillin-streptomycin mixture (GibCo BRL, Grand Island, NY, USA) at $37^{\circ} \mathrm{C}$ and
$5 \% \mathrm{CO}_{2}$. The medium was replaced $24 \mathrm{~h}$ later with fresh medium for transfection. The VEGFR2-siRNA and controlsiRNA were obtained from Life TechnologiesCo.,Ltd. The siRNA targeting VEGFR2 sequences were: 5'-CCAUGUCUCGGGUCCAUUUTTdTdT-3', 5' GCUUUACUAUUCCCAGCUAT TdTdT-3' and 5'-AAAUGGACCCGAGACAUGGTTdTdT-3'. Hep2 cells were transfected with control-siRNA or VEGFR2siRNA according to the manufacturer's instructions. Cells were collected for Western blot, CCK8 and flow cytometry assays $48 \mathrm{~h}$ after transfection.

\section{Cell cycle analyses}

For cell cycle analyses, cells were fixed in $70 \%$ ethanol overnight at $4^{\circ} \mathrm{C}$ and then incubated with $1 \mathrm{mg} / \mathrm{ml}$ RNase A for $15 \mathrm{~min}$. Subsequently, cells were stained with $0.5 \%$ Tween-20, propidiumiodide (PI, $50 \mathrm{mg} / \mathrm{ml}$, Becton Dickinson, San Jose, CA) in PBS, and analyzed with a Becton Dickinson flow cytometer BD FACScan (San Jose, CA) and Cell Quest acquisition and analysis programs. Gating was set to exclude cell debris, cell doublets, and cell clumps.

\section{MTT (Dimethyl thiazolyldiphenyltetrazolium) Assays}

At 24,48 and 72 hours after transfection, the cells were harvested for MTT assay. Hep2 cells were seeded at $5.0 \times$ 103 cells per well in the 96-well plates with five replicate wells for each condition. Every $24 \mathrm{~h}$ after transfection, $20 \mu \mathrm{l}$ MTT (Sigma, St. Louis, MO) reagent was added to respective wells, and the incubation continued for $4 \mathrm{~h}$. After the incubation, the supernatant was removed and $100 \mu \mathrm{l}$ dimethlsulfoxide (DMSO) was added to dissolve the formazan crystals. Optical density (OD) value of each sample was measured at a wavelength of $570 \mathrm{~nm}$ on an enzymed-linked immunosorbent assay plate reader (BioRad, Hercules, CA). All experiments repeated three times.

\section{In vitro migration and invasion assays}

Migration of Hep2 cells was assayed using chamber with 8 lmpore filters $(6.5 \mathrm{mmin}$ diameter, 8 lmpore size, Corning, USA). At 48 hours post-transfection, cells were plated at a density of $5.0 \times 10^{4}$ per insert in the upper chamber without serum. Medium with $20 \%$ FBS was added to the lower chamber as chemo-attractant. After incubation for $24 \mathrm{~h}$, non-invading cells were removed from the top with a cotton swab, cells that migrated to the bottom of the membrane were then fixed with $95 \%$ ethanol and stained with $0.2 \%$ crystal violet for $30 \mathrm{~min}$ at $37{ }^{\circ} \mathrm{C}$ and washed twice with PBS. Then stained cells were visualized under a microscope (high-power fields), counted in five random fields, and the average number was taken.

\section{Results}

\section{VEGFR2 express in the tumor cells of HNSCC}

Expression level of VEGFR2 in the HNSCC was examined for 109 tumor tissues and correspond paracarcinoma tissues via immunehistochemical staining. In the para-carcinoma group, VEGFR2 expressed only moderately in the endothelial cells (Figure1 A, B). On 

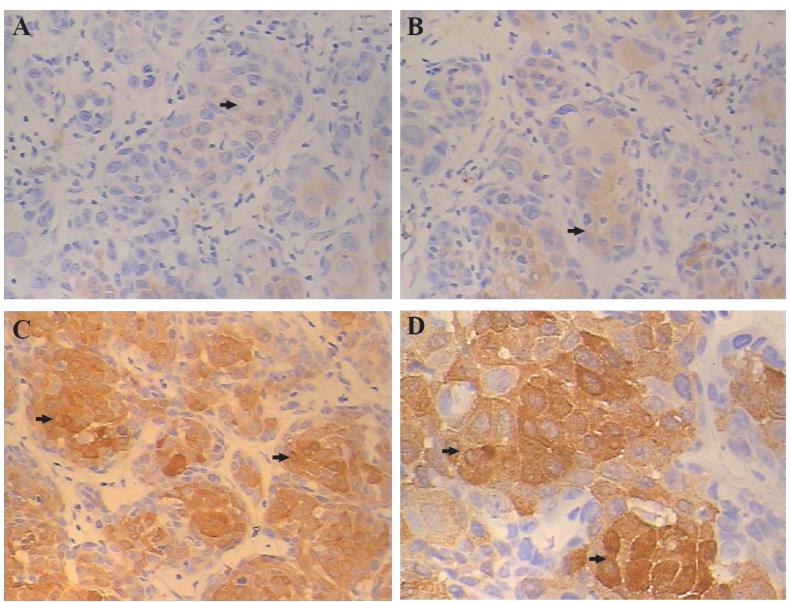

Figure 1. VEGFR2 Expresses in HNSCC Tumor Tissue and Para-carcinoma Tissues. (A, B) VEGFR2 expresses in HNSCC para-carcinoma tissues. (C, D) VEGFR2 expresses in HNSCC tumor tissue. Arrows indicate the expression cells
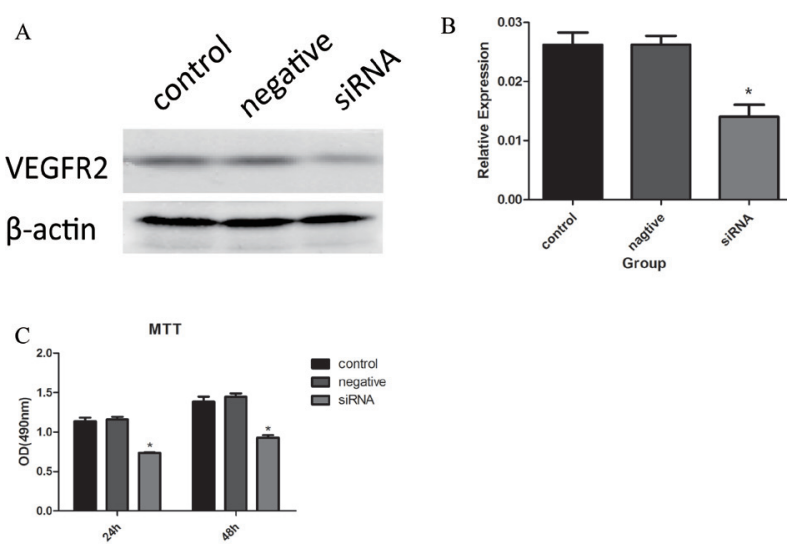

Figure 2. Knockdown of VEGFR2 can affect the Hep2 cells proliferation. $(\mathrm{A}, \mathrm{B})$ Western blot analysis shows that siRNA targeting VEGFR2 can decrease the expression of VEGFR2 $(* \mathrm{p}<0.05)$. (C) MTT assay shows that both at $24 \mathrm{~h}$ and $48 \mathrm{~h}$ after transfection of siRNA, the proliferation ability of Hep2 cells is reduced $(* \mathrm{p}<0.01)$
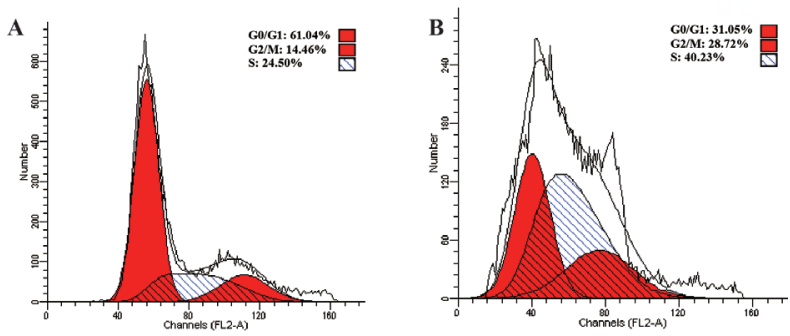

Figure 3. VEGFR2 siRNA Arrest the Cell Cycle at G0/G1. (A) The distribution of phrases of Hep2 cells treated by control siRNA (B) The distribution of phrases of Hep2 cells treated by VEGFR2 siRNA

the contrary, VEGFR2 expression was observed to be strongly and stablylocalized in both endothelial cells and surrounding tumor cells. (Figure 1 C, D)

VEGFR2 siRNA can inhibit the proliferation of Hep2 cells

To validate the function of VEGFR2 in tumor cells, we used Hep2 cells as in vitro modal. First, western blot
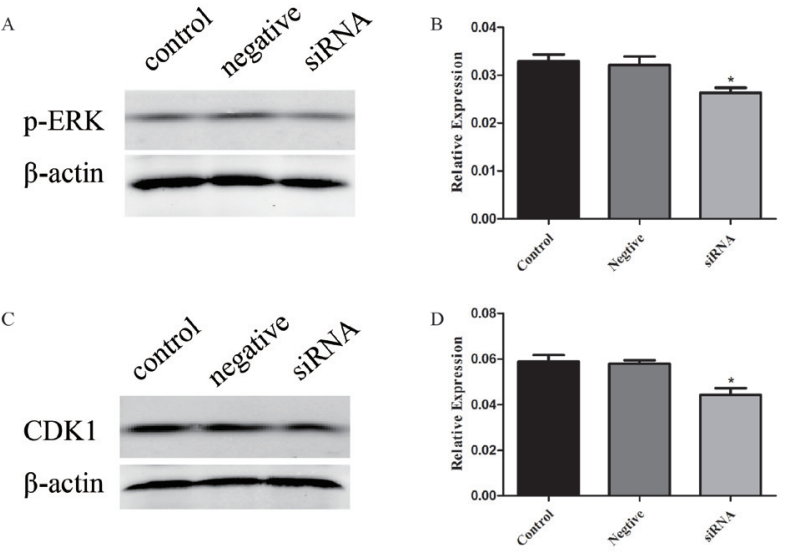

Figure 4. $p$-ERK and CDK1 act as the downstream of VEGFR2 to regulate the Hep2 proliferation. Western blot shows that the VEGFR2 siRNA significantly decreases the level of $p$-ERK $(\mathrm{A}, \mathrm{B})\left({ }^{*} p<0.05\right)$ and the expression of CDK1 $(\mathrm{C}, \mathrm{D})(* p<0.05)$
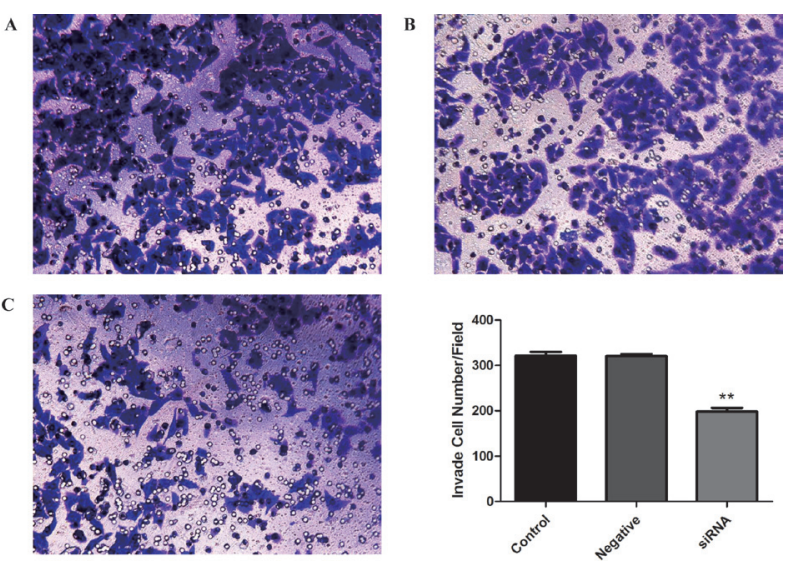

Figure 5. The Invasion Ability of Hep2 is Significantly Reduced by VEGFR2 siRNA. The Hep2 cells treated with control siRNA (B) and VEGFR2 siRNA (A) or without siRNA (C) are planted into transwell chambers to measure the immigration ability $(* * \mathrm{p}<0.01)$

was used to test the expression of VEGFR2 in Hep2 cells and the efficiency of siRNA which targeting VEGFR2. Compared with control and negative siRNA group, the expression of VEGFR2 was significantly down regulated in the group of siRNA for VEGFR2 $(p<0.05)$. (Figure 2 A, B) Then, we applied MTT colorimetry to examine the proliferation ability of Hep2 cells transfected by VEGFR2 siRNA. At both $24 \mathrm{~h}$ and $48 \mathrm{~h}$ after transfection, the absorption values for VEGFR2 siRNA group were significantly lower than the blank control group $(p<0.01)$, while there were no effects in the negative siRNA group. (Figure 2 C)

Since VEGFR2 siRNA affects proliferation, we performed flow cytometric analysis to access the cell cycle progression at 48 hours after VEGFR2 siRNA transfection of Hep2 cells. As showed in Figure 3, the percent of cells in $\mathrm{G} 0 / \mathrm{G} 1$ phrase was $61.04 \%$ in VEGFR2 siRNA group compared with $31.05 \%$ in control group. Consistently, the ratio of cells in $\mathrm{S}$ and $\mathrm{G} 2 / \mathrm{M}$ phrases drops to $24.50 \%$ and $14.46 \%$ respectively. While in siRNA group, the ratio is $40.23 \%$ and $28.72 \%$ in control group. 
ERK and CDK1 regulate the proliferation downstream of VEGFR2

To further clarify the mechanism of VEGFR2 knocking down induced anti-proliferation, we examined the expression and phosphorylation level of relating gene-CDK1 and $p$-ERK. The result of western blot demonstrated that the phosphorylation level of ERK (Figure 4 A,B)and the expression of CDK1 (Figure 4 C,D) decreased significantly $(\mathrm{P}<0.05)$.

Down-regulation of VEFGR2 leads to decrease invasion ability of Hep2 cells

Transwell assay was used to investigate the invasion ability of VEGFR2 down regulatedHep2 cells. The results showed that VEGFR2 siRNA significantly reduced the invasion of Hep2 cells compared with blank control group. While the negative control siRNA remained the same as blank group. $(p<0.01)$ (Figure 5)

\section{Discussion}

The tumorigenesis is a complex procedure, involving multiple genes and environmental factors, among which angiogenesis plays an important role in this process. If the diameter exceeds the minimal size (1-2mm), the solid tumors require a vascular stroma(Folkman and Shing, 1992).VEGFR2 was firstly identified as the receptor of vascular endothelial growth factor (VEGF) and proved to mediate the process of angiogenesis in the endothelial cells (Segiet et al., 2015). Therefore, the previous researches of VEGFR2 focused on its function of angiogenesis. However, our research revealed that VEGFR2 expressed strongly not only in the endothelial cells but also in tumor cells in HNSCC, which consisted with reports in other cancer (Guo et al., 2010; Silva et al., 2011; Mokhtari, 2012; Kontic et al., 2014). This result indicated thatVEGFR2 might play an important role inprogression of HNSCC.

There were few researches focusing on the VEGFR2's functions in cancer cells before, therefore ourstudy was designed to recognize the effect of VEGFR2 down-regulation. We used Hep2 cell line as cell model. Previously, researchers have proved that the proliferation ability wasone of key factors for tumorigenesis (Cheng et al., 2008; Kauppila et al., 2014). According to our study, MTT assay shown that the down-regulation of VEGFR2 via siRNA can significantly decreased the proliferation in Hep2 cells. In order to make clear the reason why proliferation was inhibited, we performed flow cytometric analysis. The result showed that more cells were arrested in the G0/G1phrases compared with control group. As a result, we tested the expression of CDK1 which was the key regulator of G1/S checkpoint (Corlu and Loyer, 2012), at protein level. The expression of CDK1 was significantly suppressed which may explain the cell cycle arrest. MAPK/ ERK was reported to act as the downstream of VEGFR2 to transport the proliferation signal (Schulze et al., 2005), so the phosphorylation level of ERK was determined by western blot. $p$-ERK level reduced significantly which demonstrated that MAPK/ERK signal pathway mightbe activated by VEGFR2 to mediate CDK1 expression and affect the cell cycle. It has been reported that invasion is essential in HNSCC development (Inglehart et al., 2014). Therefore, we examined invasion ability of Hep2 cells by transwell assay. VEGFR2 knockdown significantly affected Hep 2 cell invasion.

Previous researches have certified that VEGF was observed in cancer cells (Bergers and Benjamin, 2003). Autonomous VEGF synergized with EGFR signalingis essential for squamous carcinoma cell proliferation independent of angiogenesis. In this work, they identified VEGFR1 and Neuropilin-1 as the receptor for VEGF (Lichtenberger et al., 2010). Though still in debate, cancer stem cell was thought to be the causes of recurrence and metastasis. VEGF-Neuropilin 1 loop was reported to be important in cancer stemness (Beck et al., 2011). Both of them did not detect the expression of VEGFR2 in skin cancer cells. On the contrary, we observed the expression of VEGFR2 in cancer cells in HNSCC. It is possible that VEGFR2 also involves in the regulation of proliferation and invasion in the HNSCC in vivo.

VEGFR2 has been realized as the target for anticancer therapy for its function on angiogenesis (Croci and Rabinovich, 2014; Xuan et al., 2014). Our research illustrated that VEGFR2 could also mediate the proliferation and invasion in Hep2 cells which caste a light on developing anti-tumor therapy targeting on VEGFR2 not only its function on angiogenesis but also anti-proliferation and anti-invasion in HNSCC.

\section{References}

Beck B, Driessens G, Goossens S, et al (2011). A vascular niche and a VEGF-Nrp1 loop regulate the initiation and stemness of skin tumours. Nature, 478, 399-403.

Bergers G, Benjamin LE (2003). Tumorigenesis and the angiogenic switch. Nat Rev Cancer, 3, 401-10.

Brekken RA, Thorpe PE (2001). Vascular endothelial growth factor and vascular targeting of solid tumors. Anticancer Res, 21, 4221-9.

Cheng GZ, Zhang W, Wang LH (2008). Regulation of cancer cell survival, migration, and invasion by Twist: AKT2 comes to interplay. Cancer Res, 68, 957-60.

Corlu A, Loyer P (2012). Regulation of the g1/s transition in hepatocytes: involvement of the cyclin-dependent kinase cdk1 in the DNA replication. Int J Hepatol, 2012, 689324.

Croci DO, Rabinovich GA (2014). Linking tumor hypoxia with VEGFR2 signaling and compensatory angiogenesis: Glycans make the difference. Oncoimmunol, 3, 29380.

Das LC, Karrison TG, Witt ME, et al (2014). Comparison of outcomes of locoregionally advanced oropharyngeal and non-oropharyngeal squamous cell carcinoma over two decades. Ann Oncol.

Folkman J, Shing Y (1992). Angiogenesis. J Biol Chem, 267, 10931-4.

Gasparini G, Longo R, Toi M, et al (2005). Angiogenic inhibitors: a new therapeutic strategy in oncology. Nat Clin Pract Oncol, 2, 562-77.

Guo S, Colbert LS, Fuller M, et al (2010). Vascular endothelial growth factor receptor-2 in breast cancer. Biochim Biophys Acta, 1806, 108-21.

Inglehart RC, Scanlon CS, D'Silva NJ (2014). Reviewing and reconsidering invasion assays in head and neck cancer. Oral Oncol, 50, 1137-43.

Kauppila JH, Korvala J, Siirila K, et al (2014). Toll-like receptor 
9 mediates invasion and predicts prognosis in squamous cell carcinoma of the mobile tongue. J Oral Pathol Med.

Kontic M, Milovanovic J, Colovic Z, et al (2014). Epidermal growth factor receptor (EGFR) expression in patients with laryngeal squamous cell carcinoma. Eur Arch Otorhinolaryngol.

Krajewska J, Handkiewicz-Junak D, Jarzab B (2015). Sorafenib for the treatment of thyroid cancer: an updated review. Expert Opin Pharmacother, 1-11.

Lichtenberger BM, Tan PK, Niederleithner H, et al (2010). Autocrine VEGF signaling synergizes with EGFR in tumor cells to promote epithelial cancer development. Cell, 140, 268-79.

Mokhtari S (2012). Mechanisms of cyst formation in metastatic lymph nodes of head and neck squamous cell carcinoma. Diagn Pathol, 7, 6.

Parast CV, Mroczkowski B, Pinko C, et al (1998). Characterization and kinetic mechanism of catalytic domain of human vascular endothelial growth factor receptor-2 tyrosine kinase (VEGFR2 TK), a key enzyme in angiogenesis. Biochemistry, 37, 16788-801.

Pathare SM, Gerstung M, Beerenwinkel N, et al (2011). Clinicopathological and prognostic implications of genetic alterations in oral cancers. Oncol Lett, 2, 445-51.

Rehman AO, Wang CY (2009). CXCL12/SDF-1 alpha activates NF-kappaB and promotes oral cancer invasion through the Carma3/Bcl10/Malt1 complex. Int J Oral Sci, 1, 105-18.

Reya T, Clevers H (2005). Wnt signalling in stem cells and cancer. Nature, 434, 843-50.

Rothenberg SM, Ellisen LW (2012). The molecular pathogenesis of head and neck squamous cell carcinoma. J Clin Invest, 122, 1951-7.

Schulze WX, Deng L, Mann M (2005). Phosphotyrosine interactome of the ErbB-receptor kinase family. Mol Syst Biol, 1, 2005-8.

Segiet OA, Michalski M, Brzozowa-Zasada M, et al (2015). Angiogenesis in primary hyperparathyroidism. Ann Diagn Pathol.

Silva SR, Bowen KA, Rychahou PG, et al (2011). VEGFR-2 expression in carcinoid cancer cells and its role in tumor growth and metastasis. Int J Cancer, 128, 1045-56.

Xuan ZX, Li LN, Zhang Q, et al (2014). Fully human VEGFR2 monoclonal antibody $\mathrm{BC} 001$ attenuates tumor angiogenesis and inhibits tumor growth. Int J Oncol, 45, 2411-20.

Ye F, Chen HZ, Xie X, et al (2004). Vascular endothelial growth factor (VEGF) and ovarian carcinoma cell supernatant activate signal transducers and activators of transcription (STATs) via VEGF receptor-2 (KDR) in human hemopoietic progenitor cells. Gynecol Oncol, 94, 125-33. 